\title{
Autoregressive Time-Varying Flat-Fading Channels: Model Order and Information Rate Bounds
}

\author{
Parastoo Sadeghi \\ The Australian National University University of Greenwich at Medway \\ Canberra 0200 ACT Australia \\ Email: parastoo.sadeghi@anu.edu.au
}

\author{
Rodney Kennedy and Thushara Abhayapala \\ The Australian National University \\ Canberra 0200 ACT Australia \\ Email: firstname.lastname@anu.edu.au
}

\begin{abstract}
In this paper, we study the effect of channel memory order on the information rate bounds in time-varying flat-fading (FF) channels. We model time variations of the FF channel with autoregressive (AR) processes with varying degrees of model order. We observe that in high SNR conditions $(S N R \gtrsim 20 \mathrm{~dB}$ ), the information rate penalty of not knowing the AR channel is a non-increasing function of the $A R$ model order. This is expected, since the AR channel predictability cannot decrease with increasing its order. However, in low SNR conditions, the information rate penalty in low-order AR channels can be lower than those in high-order AR channels. Likewise, the intuitive and universal monotonic increase of the information rate bounds with the AR model order is only observed in almost noiseless conditions. In the low SNR regime, however, the achievable information rate bounds in low-order AR channels can be higher than those in high-order AR channels.
\end{abstract}

\section{INTRODUCTION}

\section{A. Motivation and Background}

The information capacity of time-varying fading channels has been recently investigated in the literature due to its practical significance in mobile communication systems [1][3]. The main goal of this paper is to study the effect of channel memory order on the achievable information rates in time-varying fading channels. The time-varying channel is assumed to be a random process, whose realization is unknown at the transmitter and at the receiver sides. The memory order of a random process is the number of previous realizations of the process that statistically determine its current realization. A well-known model to represent a time-varying channel process is the autoregressive (AR) model [2], [4], [5]. In an AR channel model of order $P$, hereafter denoted by $\operatorname{AR}(P)$, the current channel realization is determined from $P$ previous channel realizations and a sample from a white random process with variance $\sigma_{P}^{2}$.

A smaller variance $\sigma_{P}^{2}$ in an $\operatorname{AR}(P)$ process signifies a higher predictability of the current realization of the process from its $P$ previous realizations. From the theory of AR models, it is known that $\sigma_{P}^{2}$ is a non-increasing function of the model order $P$ [6, p. 600]. That is, for a given correlation function for the process, an $\operatorname{AR}(P+1)$ process is at least as predictable as an $\operatorname{AR}(P)$ process. In the theoretical limit, an $\mathrm{AR}(\infty)$ process with a bandlimited spectrum is deterministic, in the sense that its current realization can be predicted, with no error, given its infinite past [6, p. 600], [7].
In this paper, we aim to investigate whether the nondecreasing predictability of AR time-varying channels with the AR model order has a non-decreasing effect on the achievable information rates through the channel. From an engineering point of view, estimation and tracking of a theoretically more predictable channel could potentially be more accurate, and hence, higher information rates should be achievable at the same noisy channel observation conditions.

In an earlier work by the authors [8], it was shown that decreasing the Markov channel memory order does not necessarily decrease the capacity of the finite-state Markov channel (FSMC) [9], [10]. The capacity comparison of FSMC models with different orders showed that when the FSMC states are deeply hidden in noise, a high-order FSMC usually has a lower capacity than its low-order FSMC counterpart. The intuitive capacity increase with increasing channel memory order only happens when the FSMC states are highly observable at the receiver. Whether a similar phenomenon happens in other time-varying channels deserves further analysis.

\section{B. Approach and Contributions}

Unlike [8], which considered finite-level partitioning of the flat-fading (FF) channel gain into FSMC models, we consider continuous-level FF channels. Therefore, the findings in this paper cannot be attributed to the artifacts of finitelevel presentation of the FF channel gain in FSMC models or the choice of FF channel gain partitioning thresholds. With no channel state information (CSI) assumption, the channel capacity and the capacity-achieving input distribution for timevarying FF channels are essentially open problems [4], [11], [12]. Hence, we apply the bounds in [12], [13] to our problem to study the achievable information rate bounds in AR-FF channels.

The contributions of this paper are summarized as follows.

1) In low SNR conditions, the information rate penalty due to not knowing a high-order AR channel is higher than the penalty due to not knowing its low-order AR channel counterpart (Fig. 1, SNR $\lesssim 7 \mathrm{~dB}$ ). In fact, higher predictability of higher-order AR channels only has a non-increasing effect on the information rate penalty in very high SNR conditions.

2) The information rate bounds in AR channels also exhibit a non-monotonic behavior with the channel memory 
order in the low SNR regime. Our analysis shows that in the low SNR regime, the achievable information rate bounds in low-order AR channels are often higher than those in high-order AR channels (Fig. 2, SNR $\lesssim 7 \mathrm{~dB}$ and Fig. 4, SNR $\lesssim 10 \mathrm{~dB}$ ). The universal monotonic increase of the information rate bounds with AR model order and its predictability only happens in almost noiseless conditions.

\section{SySTEM MODEL}

With the FF channel assumption, the low-pass received signal at the discrete time index $k$ is

$$
y_{k}=h_{k} x_{k}+n_{k},
$$

where $x_{k}$ is the transmitted signal, $h_{k}$ is the complex-valued and Gaussian-distributed FF channel gain, and $n_{k}$ is a sample of complex-valued, additive white Gaussian noise (AWGN). Furthermore, we assume that $h_{k}$ has a zero mean and a normalized variance of 0.5 per dimension and $n_{k}$ has a zero mean and variance of $N_{0} / 2$ per dimension. The average transmitted power and SNR per symbol are denoted as $\mathcal{E}_{s}=E\left\{\left|x_{k}\right|^{2}\right\}$ and $\gamma_{s}=\mathcal{E}_{s} / N_{0}$, respectively.

We further assume that the time-varying FF channel gain $h_{k}$ is related to its past realizations through the $\operatorname{AR}(P)$ model

$$
h_{k}=-\sum_{p=1}^{P} a_{p} h_{k-p}+w_{k},
$$

where $w_{k}$ is a complex-valued, white Gaussian noise with variance $\sigma_{P}^{2}$ and the vector $\mathbf{a}=\left[a_{1}, \cdots, a_{P}\right]^{T}$ contains the AR filter coefficients and is derived from the Yule-Walker equation [14, pp. 55-57]:

$$
\mathbf{R a}=-\mathbf{v},
$$

where $\mathbf{R}$ is the $P \times P$ Toeplitz covariance matrix of the process, whose element at row $i$ and column $j$ only depends on $|i-j|$ and is denoted as $R(|i-j|)$. The vector $\mathbf{v}$ is given as

$$
\mathbf{v}=[R(1), R(2), \cdots, R(P)]^{T} .
$$

If we assume that the FF channel follows the Clarke's model [15, Ch. 14], then $R(|i-j|)$ is given as

$$
R_{\text {Clarke }}(|i-j|)=J_{0}\left(2 \pi f_{D}|i-j|\right),
$$

where $J_{0}$ is the zero-order Bessel function of the first kind and $f_{D}$ is the Doppler frequency shift that is normalized by the transmitted symbol period. Alternatively, for the ease of spectral analysis in Section IV, we may assume that $R(|i-j|)$ is given as

$$
R_{\mathrm{U}}(|i-j|)=\operatorname{sinc}\left(2 \pi f_{D}|i-j|\right),
$$

which is, in fact, equivalent to 'the Clarke's model' for $3 \mathrm{D}$ isotropic scattering. In an $\operatorname{AR}(P)$, the complete set of covariance elements $R_{h}(n)$ is given as [16]

$$
R_{h}(n)=\left\{\begin{array}{cl}
R(n) & |n| \leqslant P \\
-\sum_{p=1}^{P} a_{p} R_{h}(n-p) & |n|>P
\end{array},\right.
$$

where $R(n)$ for $|n| \leqslant P$ was defined in (5) or (6). The power spectral density (PSD) of $\mathrm{AR}(P)$ is a rational function

$$
H_{P}(\omega)=\frac{\sigma_{P}^{2}}{\left|1+\sum_{p=1}^{P} a_{p} e^{-j p \omega}\right|^{2}}
$$

For the special case of $\operatorname{AR}(\infty)$, we obtain the well-known bowl-shaped PSD for the Clarke's model, which is given as

$$
H_{\text {Clarke }}(\omega)=\left\{\begin{array}{cc}
\frac{1}{\pi f_{D} \sqrt{1-\left(\omega / 2 \pi f_{D}\right)^{2}}} & |\omega|<2 \pi f_{D} \\
0 & \text { otherwise }
\end{array},\right.
$$

and a uniform PSD for $R_{\mathrm{U}}$ in (6) as

$$
H_{\mathrm{U}}(\omega)=\left\{\begin{array}{cc}
\frac{1}{2 f_{D}} & |\omega|<2 \pi f_{D} \\
0 & \text { otherwise }
\end{array} .\right.
$$

The variance $\sigma_{P}^{2}$ of the white noise $w_{k}$ in (2) is the minimum mean square error (MMSE) of the one-step predictor of order $P$ for the fading process $h_{k}$ [6, pp. 598-601] and is given as

$$
\sigma_{P}^{2}=\exp \left(\frac{1}{2 \pi} \int_{-\pi}^{\pi} \ln H_{P}(\omega) d \omega\right)
$$

where ln stands for natural logarithm. It is known that the MMSE is a non-increasing function of the prediction order $P$ [6, p. 600]. That is,

$$
\sigma_{P+1}^{2} \leqslant \sigma_{P}^{2}, \quad \forall P .
$$

In other words, the $\operatorname{AR}(P)$ channel is at most as equally predictable as the $\mathrm{AR}(P+1)$ channel. From (11), we observe that if the asymptotic PSD of the fading process is zero over some frequency interval, the asymptotic MMSE prediction error is zero. In other words, a bandlimited fading process (as in (9) or (10)) is, in theory, deterministic in the sense that its future realization can be predicted from its infinite past with no error [7].

Now, reconsider the unknown FF channel with observation equation (1), where the $\mathrm{FF}$ channel gain varies with time according to the $\operatorname{AR}(P)$ model in (2). From the predictability discussion following (11)-(12), one might be inclined to conclude that the achievable information rates in an $\operatorname{AR}(P)$ channel are generally lower than those in an $\operatorname{AR}(P+1)$ channel. In the limit, it might be even conjectured that an $\operatorname{AR}(\infty)$ model with the highest predictability has the highest achievable information rate among its lower-order and less predictable AR counterparts. However, the predictability analysis in (11)-(12) lies on the important assumption that the channel's infinite past is already observed without error and is available. This is equivalent to assuming a noiseless channel observation equation in (1) and a known transmitted signal $\left(N_{0}=0\right.$ and known $x_{k}$ ). The analysis in Section IV reveals, however, that in low to medium SNR conditions, when the channel realizations are not directly observable, the effect of increasing channel memory order on the information rate bounds is not monotonic with the AR model order. 


\section{INFORMATION RATE BOUNDS}

The information capacity and the capacity-achieving input distribution of temporally correlated, time-varying FF channels with no CSI at the transmitter and at the receiver are generally open problems [3], [4], [11], [12]. Therefore, we limit ourselves to analyze and compare the information rate upper and lower bounds in correlated FF channels with varying degrees of AR model order. We start by briefly reviewing the information rate bounds in [12]. The advantage of these bounds is that they have closed-form and single-letter expressions and provide valuable insight into our problem. Later in Section IV, we will verify our observations by using tighter bounds in [13], which can only be numerically computed. All mutual information rates are computed in nats per channel use.

Let $\mathbf{x}, \mathbf{y}$, and $\mathbf{h}$ be $N \times 1$ sequences of transmitted signal, received signal, and channel realization, respectively. Using the chain rule on the mutual information $I(\mathbf{y} ; \mathbf{x}, \mathbf{h})$, we can write the mutual information between $\mathbf{y}$ and $\mathbf{x}$ as

$$
\begin{aligned}
I(\mathbf{y} ; \mathbf{x}) & =I(\mathbf{y} ; \mathbf{x}, \mathbf{h})-I(\mathbf{y} ; \mathbf{h} \mid \mathbf{x}) \\
& =I(\mathbf{y} ; \mathbf{h})+I(\mathbf{y} ; \mathbf{x} \mid \mathbf{h})-I(\mathbf{y} ; \mathbf{h} \mid \mathbf{x}),
\end{aligned}
$$

where $I(\mathbf{y} ; \mathbf{x} \mid \mathbf{h})$ is the mutual information with perfect CSI and $I(\mathbf{y} ; \mathbf{h} \mid \mathbf{x})-I(\mathbf{y} ; \mathbf{h})$ is the penalty in mutual information when the CSI is not available. Since $I(\mathbf{y} ; \mathbf{h}) \geqslant 0$, it is concluded that $I(\mathbf{y} ; \mathbf{h} \mid \mathbf{x})$ is an upper bound on the mutual information penalty due to unknown CSI. An upper bound on $I(\mathbf{y} ; \mathbf{h} \mid \mathbf{x})$ can be derived as (see [12] for more details)

$$
I(\mathbf{y} ; \mathbf{h} \mid \mathbf{x}) \leqslant \sum_{n=1}^{N} \ln \left(1+\frac{\mathcal{E}_{s}}{N_{0}} \lambda_{n}\right)
$$

where $\left\{\lambda_{n}\right\}_{n=1}^{N}$ are the eigenvalues of the $N \times N$ Toeplitz covariance matrix of channel vector $\mathbf{h}$ with elements defined in (7). The asymptotic information rate per symbol for $N \rightarrow \infty$ is then upper bounded as

$$
\begin{aligned}
\Delta_{P} & \triangleq \mathcal{I}_{P}(y ; h \mid x)=\lim _{N \rightarrow \infty} \frac{1}{N} I(\mathbf{y} ; \mathbf{h} \mid \mathbf{x}) \\
& \leqslant \frac{1}{2 \pi} \int_{-\pi}^{\pi} \ln \left(1+\frac{\mathcal{E}_{s}}{N_{0}} H_{P}(\omega)\right) d \omega .
\end{aligned}
$$

The equality in (17) is achieved for constant power signaling such as $M$-ary PSK schemes [15, Ch. 4-5]. Therefore, it is possible to upper bound $I(\mathbf{y} ; \mathbf{x})$ for constant power signaling by using (13) and upper bounding $I(\mathbf{y} ; \mathbf{h}, \mathbf{x})=$ $H(\mathbf{y})-H(\mathbf{y} \mid \mathbf{h}, \mathbf{x})=H(\mathbf{y})-H(\mathbf{n})$ by assuming a Gaussian distribution for $\mathbf{y}$. The following asymptotic information rate upper bound is derived for constant power signaling

$$
\begin{aligned}
\mathcal{I}_{P}^{C}(y ; x) & \leqslant \ln \left(1+\frac{\mathcal{E}_{s}}{N_{0}}\right)-\Delta_{P} \\
& =\ln \left(1+\frac{\mathcal{E}_{s}}{N_{0}}\right)-\frac{1}{2 \pi} \int_{-\pi}^{\pi} \ln \left(1+\frac{\mathcal{E}_{s}}{N_{0}} H_{P}(\omega)\right) d \omega .
\end{aligned}
$$

Using the fact that $I(\mathbf{y} ; \mathbf{h})$ is non-negative in (14), we obtain the following lower bound on $I(\mathbf{y} ; \mathbf{x})$

$$
I(\mathbf{y} ; \mathbf{x}) \geqslant I(\mathbf{y} ; \mathbf{x} \mid \mathbf{h})-I(\mathbf{y} ; \mathbf{h} \mid \mathbf{x}) .
$$

In order to evaluate the asymptotic information rate per symbol, we use (16)-(17) and the fact that the mutual information rate with CSI is independent of time index. This yields

$$
\begin{aligned}
\mathcal{I}(y ; x \mid h) & =H(y \mid h)-H(y \mid x, h)=H(y \mid h)-H(n) \\
& =H(y \mid h)-\ln \left(\pi e N_{0}\right),
\end{aligned}
$$

and therefore,

$$
\begin{aligned}
\mathcal{I}(y ; x) & \geqslant \mathcal{I}(y ; x \mid h)-\Delta_{P} \\
& \geqslant \mathcal{I}(y ; x \mid h)-\frac{1}{2 \pi} \int_{-\pi}^{\pi} \ln \left(1+\frac{\mathcal{E}_{s}}{N_{0}} H_{P}(\omega)\right) d \omega .
\end{aligned}
$$

For $M$-ary PSK signaling, the CSI entropy rate $H(y \mid h)$ only depends on the channel gain amplitude $r=|h|$ and is given as

$$
H(y \mid h)=\int_{0}^{\infty} \int_{-\infty}^{\infty} f(y \mid r) f(r) \ln f(y \mid r) d y d r
$$

where $f(y \mid r)$ is the conditional pdf of $y$ given $r$ and $r$ is Rayleigh-distributed. For equiprobable, constant power, $M$ ary PSK signaling $f(y \mid r)$ is given by

$$
f(y \mid r)=\frac{1}{M} \sum_{m=0}^{M-1} f\left(y \mid x_{m}, r\right),
$$

where $f\left(y \mid x_{m}, r\right)$ is the pdf of AWGN in (1) with mean $r x_{m}=r \sqrt{\mathcal{E}_{s}} e^{j 2 \pi m / M}$ and variance $N_{0} / 2$ per dimension.

\section{ANALYSis OF INFORMATION RATE BOUndS}

We first examine the information rate upper and lower bounds in (18) and (21) in more detail. We observe that the first terms in (18) and (21) are independent of the dynamics of time-varying AR channel and only depend on SNR. On the other hand, the second term $\Delta_{P}$, given in (17) depends on both the $\operatorname{AR}(P)$ spectrum $H_{P}(\omega)$ and $\mathrm{SNR} \mathcal{E}_{s} / N_{0}$.

The information rate penalty $\Delta_{P}$ for the special case of $\mathrm{AR}(1)$ has a closed form. To see this, we use (3) to rewrite the AR spectrum in (8) as

$$
\begin{aligned}
H_{1}(\omega) & =\frac{\sigma_{1}^{2}}{\left|1+a_{1} e^{-j \omega}\right|^{2}} \\
& =\frac{1-R^{2}(1)}{1+R^{2}(1)-2 R(1) \cos \omega} .
\end{aligned}
$$

Upon using (25) in (17) and the integral identity [17, p. 526]

$$
\int_{-\pi}^{\pi} \ln (1+a \cos x) d x=2 \pi \ln \frac{1+\sqrt{1-a^{2}}}{2},
$$

we obtain

$$
\Delta_{1}=\ln \frac{A+\sqrt{A^{2}-B^{2}}}{C+\sqrt{C^{2}-B^{2}}},
$$

where $A \triangleq 1+R^{2}(1)+\mathcal{E}_{s} / N_{0}\left(1-R^{2}(1)\right), B \triangleq 2 R(1)$, and $C \triangleq 1+R^{2}(1)$.

The information rate penalty $\Delta_{\infty}$ for $\operatorname{AR}(\infty)$ with the uniform spectrum (10) is simply derived to be

$$
\Delta_{\infty}=2 f_{D} \ln \left(1+\frac{\mathcal{E}_{s}}{N_{0}} \frac{1}{2 f_{D}}\right) .
$$




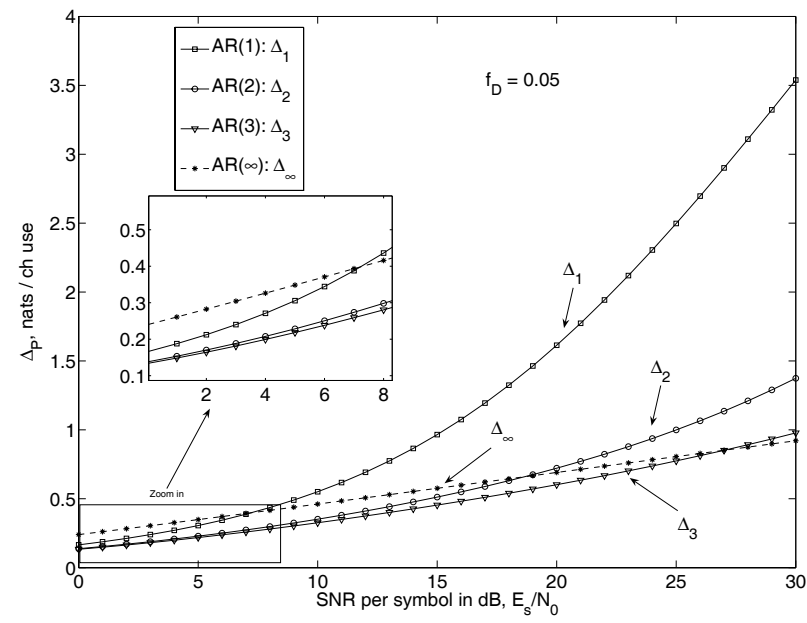

Fig. 1. The information rate penalty due to unknown CSI $\Delta_{P}$, given in (17), as a function of SNR and the AR channel model order $P$. As shown in the enlarged graphs, for SNR $\lesssim 7 \mathrm{~dB}$, the penalty in information rate due to unknown CSI is highest in the most "ideally predictable" channel model $\operatorname{AR}(\infty)$. In fact, higher channel predictability has the expected monotonically decreasing effect on the information rate penalty only in very high SNR (ideally noiseless) conditions. This is observed for SNR $\gtrsim 27 \mathrm{~dB}$.

The information rate penalty $\Delta_{P}$ for $P>1$ is numerically evaluated by directly using (8) in (17).

Fig. 1 shows the information rate penalty $\Delta_{P}$ at the normalized fading rate of $f_{D}=0.05$. The elements of $\mathbf{R}$ in (3) are chosen from (6) (uniform fading spectrum). Four different AR models with $P=1$ to $P=3$ and $P=\infty$ are shown. From this figure, it is clearly observed that the information rate penalty $\Delta_{P}$ due to unknown CSI does not necessarily decrease with increasing the channel memory order (increasing channel predictability). For example, the penalty in the achievable information rate in an $\operatorname{AR}(\infty)$ channel, $\Delta_{\infty}$, is larger than the penalty in the achievable information rate in an $\mathrm{AR}(1)$ channel, $\Delta_{1}$, for $\mathcal{E}_{s} / N_{0} \lesssim 7 \mathrm{~dB}$. This is despite the fact that $\mathrm{AR}(1)$ is the least predictable channel according to (12). Similarly, $\Delta_{\infty}>\Delta_{3}$ for $\mathcal{E}_{s} / N_{0} \lesssim 27 \mathrm{~dB}$. Only in very high SNR conditions, do we observe a monotonically decreasing information rate penalty with increasing $P$. In fact, this phenomenon can be qualitatively explained by referring to the integrand in (17). In very high SNR conditions, the constant term 1 in the logarithm is negligible, compared to the second term and may be ignored. In this case, the behavior of $\Delta_{P}$ with memory order would be very much like the behavior of $\sigma_{P}^{2}$ in (11), which is non-increasing as $P$ grows large. However, in low to medium SNR conditions, the constant term in the integrand in (17) may not be ignored and the real behavior of $\Delta_{P}$ depends on $\mathrm{SNR}$ and $\operatorname{AR}(P)$ spectrum. The corresponding information rate upper bounds for constant power signaling, $\mathcal{I}_{P}^{C}$ in (18), are shown in Fig. 2. It is verified that the non-monotonic behavior of $\Delta_{P}$ translates into nonmonotonic information rate upper bounds. For example, the information rate upper bound in the $\operatorname{AR}(3)$ channel is higher

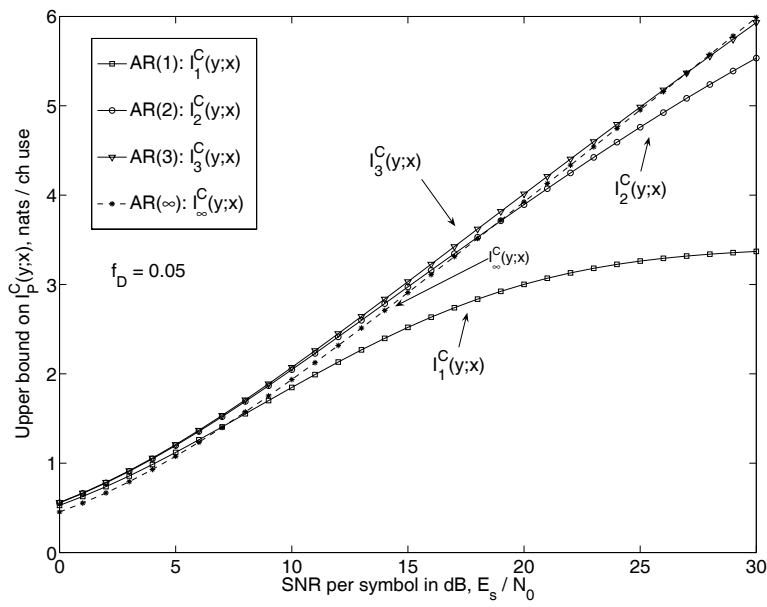

Fig. 2. The non-monotonic behavior of the information rate penalty with the AR model order in Fig. 1 is translated into the non-monotonic behavior of information rate upper bounds for constant power signaling. For example, the information rate upper bound in the $\operatorname{AR}(\infty)$ channel, $\mathcal{I}_{\infty}^{C}(y ; x)$ is lower than $\mathcal{I}_{3}^{C}(y ; x)$ in the $\mathrm{AR}(3)$ channel for SNR conditions $\mathcal{E}_{s} / N_{0} \lesssim 27 \mathrm{~dB}$.

than that in the $\operatorname{AR}(\infty)$ channel for $\mathcal{E}_{s} / N_{0} \lesssim 27 \mathrm{~dB}$.

In Fig. 3, we have shown the information rate upper bounds for constant power signaling, $\mathcal{I}_{P}^{C}$ in $(18)$, as a function of the normalized Doppler frequency shift $f_{D}$ for two low SNR conditions of $\mathcal{E}_{s} / N_{0}=0$ and $\mathcal{E}_{s} / N_{0}=3 \mathrm{~dB}$ and two $\mathrm{AR}$ models $\operatorname{AR}(1)$ and $\operatorname{AR}(\infty)$. The elements of $\mathbf{R}$ in (3) are chosen from (6) (uniform fading spectrum). $\mathcal{I}_{1}^{C}$ and $\mathcal{I}_{\infty}^{C}$ both have closed-form expressions according to (18) and (27)-(28). From this figure it is observed that $\mathcal{I}_{1}^{C}$ in $\operatorname{AR}(1)$ is higher than $\mathcal{I}_{\infty}^{C}$ in $\operatorname{AR}(\infty)$ for slow to medium-speed fading rates. However, for very fast fading rates $\mathcal{I}_{\infty}^{C}$ is higher. It is also verified from this figure that $\mathcal{I}_{\infty}^{C}$ is higher than $\mathcal{I}_{1}^{C}$ for a wider range of fading rates at higher SNR conditions. As expected, when the SNR is sufficiently high $\left(\mathcal{E}_{s} / N_{0} \gtrsim 10 \mathrm{~dB}\right), \mathcal{I}_{\infty}^{C}$ is universally higher than $\mathcal{I}_{1}^{C}$ for almost all fading rates. This is not shown in Fig. 3 for more clarity of the figure.

In Fig. 4, we have shown the information rate lower bounds in (21) by assuming QPSK constant power signaling. The normalized fading rate is $f_{D}=0.01$. The bounds tend to become loose in high SNR conditions. The reason is that in (21), the first term is specifically calculated for QPSK and hence, $\mathcal{I}(y ; x \mid h) \leqslant 2 \ln 2=1.39$ nats/ch use, whereas the second term in (21) generally holds for all constant power signaling schemes and becomes large for high SNR. Nevertheless, the behavior of the lower bound at low SNR conditions is noteworthy. In fact, the information lower bounds in $\mathrm{AR}(1)$ to $\mathrm{AR}(3)$ models are all higher than that of $\mathrm{AR}(\infty)$ for $\mathcal{E}_{s} / N_{0} \lesssim 10 \mathrm{~dB}$. At higher SNR conditions, the information rate lower bound in $\operatorname{AR}(3)$ is still higher than that in $\operatorname{AR}(\infty)$.

We have also computed the lower and upper bounds in [13] for two $\operatorname{AR}(1)$ and $\operatorname{AR}(\infty)$ channels. The bounds are tighter than those in [12], but can only be numerically computed. The upper bound in [13] can be computed for constant power 


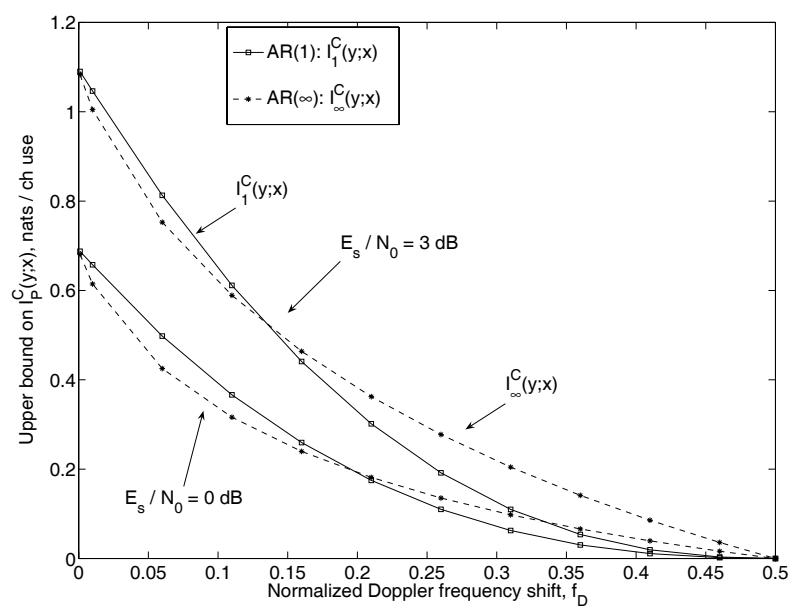

Fig. 3. The information rate upper bounds, $\mathcal{I}_{P}^{C}$ in (18), for constant power signaling as a function of the normalized Doppler frequency shift. In very fast fading rates, $\mathcal{I}_{\infty}^{C}$ is higher than $\mathcal{I}_{1}^{C}$. In relatively slow to medium-speed fading conditions, $\mathcal{I}_{1}^{C}$ is higher. The transition point depends on the SNR. In lower SNR conditions, $\mathcal{I}_{1}^{C}>\mathcal{I}_{\infty}^{C}$ for a wider range of fading rates.

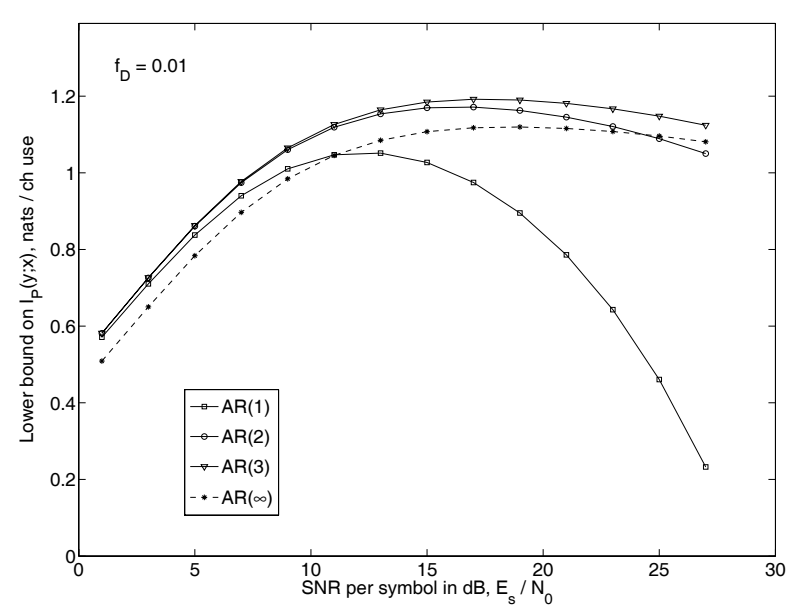

Fig. 4. The information rate lower bounds for QPSK signaling, given in (21), as a function of SNR. The information rate lower bounds in $\operatorname{AR}(1)$ to $\operatorname{AR}(3)$ channels are all higher than that in the $\operatorname{AR}(\infty)$ channel for $\mathcal{E}_{s} / N_{0} \lesssim 10 \mathrm{~dB}$. The information rate lower bound in the $\operatorname{AR}(3)$ channel is higher than that in the $\operatorname{AR}(\infty)$ channel for all considered SNR values.

signaling, since $H_{P}(y \mid x)=\mathcal{I}_{P}(y ; h \mid x)+H_{P}(y \mid x, h)=\Delta_{P}+$ $H(n)=\Delta_{P}+\ln \left(\pi e N_{0}\right)$. For the fading rate $f_{D}=0.05$, BPSK scheme, and SNR of $0 \mathrm{~dB}$, the information rate lower bound in [13] for the $\operatorname{AR}(1)$ channel yields $\underline{I}_{1}=0.3062$ nats/ch use, whereas for the $\operatorname{AR}(\infty)$ channel $\underline{I}_{\infty}=0.2361$ nats/ch use. The upper bound for the $\operatorname{AR}(1)$ channel yields $\bar{I}_{1}=0.3830$ nats/ch use, whereas for the $\operatorname{AR}(\infty)$ channel $\bar{I}_{\infty}=0.3308$ nats/ch use. The upper bounds in Fig. 2 for this SNR were $I_{1}^{C}=0.5275$ and $I_{\infty}^{C}=0.4534$ nats/ch use, respectively. This confirms the non-increasing behavior of information rates with increasing the AR model order at low SNR values.
We also observed that similar conclusions as in Figs. 1-4 can be drawn by using the Clarke's spectrum in (9), instead of (10). Therefore, the numerical results are omitted here.

\section{CONCLUSIONS}

The theoretical higher channel predictability of high-order AR channel models is only well-defined in almost noiseless conditions (high SNR regime). In this case, one can expect that the achievable information rates in time-varying channels are non-decreasing functions of the channel memory order. On the other hand, in the low SNR regime, higher channel memory order and ideal channel predictability do not affect the achievable information rates as expected. Our analysis showed that in many low SNR conditions and for a wide range of channel fading rates, the information rate bounds in low-order AR channels are higher than those in high-order AR channels. Our analysis is in accordance with the findings in [8] on the non-monotonic effect of memory order on the FSMC capacity.

\section{REFERENCES}

[1] E. Biglieri, J. Proakis, and S. Shamai, "Fading channels: Informationtheoretic and communications aspects," IEEE Trans. Inform. Theory, vol. 44, no. 6, pp. 2619-2692, Oct. 1998.

[2] M. Medard, "The effect upon channel capacity in wireless communications of perfect and imperfect knowledge of the channel," IEEE Trans. Inform. Theory, vol. 46, no. 3, pp. 933-946, May 2000.

[3] Y. Liang and V. V. Veeravalli, "Capacity of noncoherent time-selective Rayleigh-fading channels," IEEE Trans. Inform. Theory, vol. 50, no. 12, pp. 3095-3110, Dec. 2004.

[4] J. Zhang;, "On the bounds of the non-coherent capacity of GaussMarkov fading channels," in Proc. IEEE Int. Conf. on Acoustic, Speech, and Signal Processing (ICASSP), Mar. 2005, pp. 757-760.

[5] R. Chen, B. Hajek, R. Koetter, and U. Madhow, "On fixed input distributions for noncoherent communication over high-SNR Rayleighfading channels," IEEE Trans. Inform. Theory, vol. 50, no. 12, pp. 3390 3396, Dec. 2004.

[6] A. Papoulis and S. U. Pillai, Probability, Random Variables and Stochastic Processes, 4th ed. New York: Mc Graw Hill, 2002.

[7] K. E. Baddour and N. C. Beaulieu, "Autoregressive models for fading channel simulation," in IEEE Global commun. Conf. (GLOBECOM), San Antonio, TX, Nov. 2001, pp. 1187-1192.

[8] P. Sadeghi, P. B. Rapajic, and Z. B. Krusevac, "The effect of memory order on the capacity of finite-state markov and flat-fading channels," in Proc. IEEE Int. Symp. on Inform. Theory (ISIT), Adelaide, Australia, Sept. 2005, pp. 525-529.

[9] R. G. Gallager, Information Theory and Reliable Communication. New York: Wiley, 1968.

[10] A. J. Goldsmith and P. Varaiya, "Capacity, mutual information, and coding for finite-state Markov channels," IEEE Trans. Inform. Theory, vol. 42, no. 3, pp. 868-886, May 1996.

[11] I. C. Abou-Faycal, M. D. Trott, and S. Shamai, "The capacity of discretetime memoryless Rayleigh-fading channels," IEEE Trans. Inform. Theory, vol. 47, no. 4, pp. 1290-1301, May 2001.

[12] X. Deng and A. M. Haimovich, "Information rates of time varying Rayleigh fading channels," in IEEE Int. Conf. Commun.(ICC), Paris, France, June 2004, pp. 573-577.

[13] D. Arnold, H. A. Loeliger, and P. O. Vontobel, "Computation of information rates from finite-state source/channel models," in Proc. 40th Allerton Conf. on Commun., Control, and Computing, Monticello, Illinois, Oct. 2002.

[14] S. Haykin, Adaptive Filter Theory, 4th ed. Upper Saddle River: Prentice Hall, 2002.

[15] J. G. Proakis, Digital Communications, 4th ed. New York: Mc Graw Hill, 2000.

[16] S. M. Kay, Modern Spectral Estimation. Englewood Cliffs, NJ: Prentice Hall, 1988.

[17] I. S. Gradshteyn and I. M. Ryzhik, Table of Integrals, Series, and Products, 6th ed. San Diego: Academic Press, 2000. 